\title{
Laser Processing of Sub-Wavelength Structures on Sapphire and Alu- mina for Millimeter Wavelength Broadband Anti-Reflection Coatings
}

\author{
Viktor Schütz*, Karl Young***, Tomotake Matsumura**, Shaul Hanany***, Jürgen Koch*, Oliver Suttmann*, \\ Ludger Overmeyer*, and Qi Wen*** \\ * Laser Zentrum Hannover e.V., Hollerithallee 8, 30419 Hannover, Germany \\ E-mail:v.schuetz@lzh.de \\ ** Japan Aerospace Exploration Agency (JAXA) - Institute of Space and Astronautical Science \\ (ISAS), Rm1624, 3-1-1 Yoshinodai, Chuo, Sagamihara, Kanagawa 252-5210, Japan \\ *** School of Physics and Astronomy and Minnesota Institute for Astrophysics, University of Min- \\ nesota/Twin Cities, 116 Church St. SE Minneapolis, MN 55455, USA
}

\begin{abstract}
Alumina, sapphire and silicon optical elements are used for the detection of cosmic microwave background radiation with its peak intensity between 0.5 and $10 \mathrm{~mm}$. The reflectivity of such material is high but can be reduced with sub-wavelength structures acting as an anti-reflective coating. Required structure height of over $1 \mathrm{~mm}$ can be produced by laser machining. Using ultra-short laser pulses we machined $>1 \mathrm{~mm}$ high pyramidal structures on sapphire and alumina. The laser process has been optimized to achieve high area structuring speeds for $1 \mathrm{~mm}$ high structures, up to $425 \mathrm{~mm}^{2} / \mathrm{h}$. The structured surfaces have been analysed with secondary-electron microscopy, optical confocal microscopy, and transmission spectroscopy in the $100 \mathrm{GHz}$ range and provide an antireflection coating for optical elements in the millimeter wave-length.
\end{abstract}

DOI: $10.2961 / \mathrm{jlmn} .2016 .02 .0011$

Keywords: Ultra short pulsed laser processing, anti-reflection, cosmic-microwave background, sapphire, alumina

\section{Introduction}

In the millimeter wave-band alumina, sapphire and silicon have an index of refraction $n \sim 3$ and are therefore useful as optical components. For example, several experiments that measure properties of the cosmic microwave background radiation (CMB), which has its peak intensity between 0.5 and $10 \mathrm{~mm}$, use these materials [1-4]. But the high index also requires an anti-reflection coating (ARC) to reduce the $\sim 30 \%$ reflection per surface. An effective ARC is provided with a single $\lambda / 4$ thick layer that has an index of $\sqrt{n}$, but this ARC is useful over only $\sim 25 \%$ bandwidth. Patterning the surface of optical elements with subwavelength structures (SWS) gives an ARC with significantly broader bandwidths. Southwell showed that bandwidths up to $100 \%$ can be achieved [5]. An ARC made with SWS is also beneficial because it is more robust in cryogenic applications, as is the case with many CMB instruments.

Datta et al. describe patterning of silicon with SWS using commercially available dicing saws [6]. This technique extends the mechanical machining used by Hanany and his collaborators on rexolite [7]. But sapphire and alumina have Knoop hardness that is $>2$ and 2 times larger than rexolite and silicon, respectively, and thus not amenable to mechanical machining.

In this paper we study the structuring of sapphire and alumina using ablation with ultra-short pulsed laser radiation. With laser ablation a wide variety of shapes can be produced, including shapes without sharp corners such as the one demonstrated by Datta et al [6]. Furthermore, there is a promise for better shape-repeatability because there is no wear and tear of the tool throughout the process.
Detailed investigation of ablation parameters is necessary to achieve efficient machining. This is particularly important for large optical elements, exceeding few $\mathrm{cm}$ in diameter, for which the combination of SWS depth and machined area can make ablation times prohibitively long.

In this paper we report on fabrication of SWS on alumina and sapphire. We also describe how the geometric parameters of the ablated shapes and the ablation rate depend on laser parameters.

\section{Experimental setup}

\subsection{Sample Characteristics and Shape requirements}

We ablate one side of $50 \mathrm{~mm}$ diameter alumina and ccut sapphire disks. The pre-ablated disks are 2-4 mm thick. Alumina is sintered aluminum oxide, while sapphire is a single crystal. The index of refraction in the visible waveband, as appropriate for our laser system, is approximately 1.77 [8].

The shape of the SWS designed for this study is shown in Fig. 1 and Tab. 1 gives the design values of the parameters.

Table 1 Design parameters for structured samples

\begin{tabular}{cccc}
\hline Sample & $\begin{array}{c}\text { Height } \\
\text { in } \mu \mathrm{m}\end{array}$ & $\begin{array}{c}\text { Pitch } \\
\text { in } \mu \mathrm{m}\end{array}$ & $\begin{array}{c}\text { Peak width } \\
\text { in } \mu \mathrm{m}\end{array}$ \\
\hline All & 810 & 330 & 60 \\
\hline
\end{tabular}

The aspect ratio, defined as $a=h / p$, is a useful measure of the difficulty of machining narrow and tall structures, as are needed for broadband ARC. The aspect ratio for the current SWS is at maximum 3.3 but ultimately aspect ratios near $a=7$ are required to provide an ARC over 
the desired $300 \mathrm{GHz}$ of bandwidth between $\sim 50$ and 350 $\mathrm{GHz}$.

\subsection{Estimation of ablated shape}

Ablation of materials with ultra-short laser pulses can be in general understood with the two temperature model (TTM) [9-10]. In this model different temperatures are associated with the electron- and phonon-systems of the processed material. The model appropriately describes ablation with a single laser pulse [11]. However, for the current task this approach is computationally demanding because it would require approximately 451 thousand pulses to simulate the fabrication of the unit cell of the pyramidal structure.

Schütz et al. proposed using a state function model for laser ablation [12]; this technique is more appropriate here as it drastically reduces the amount of calculations necessary. Using this model we estimate the maximum structure height $h$, measured relative to the originally planar surface that can be achieved given beam focus position and fluence $\Phi_{\text {pro }}$. We project the beam onto a sloped surface that is tilted with a known flank angle $\alpha_{\mathrm{fl}}$ and find the depth at which the fluence no longer exceeds the ablation threshold. This gives the maximum height $h$. Figure 1 illustrates the approach.

\section{a)}

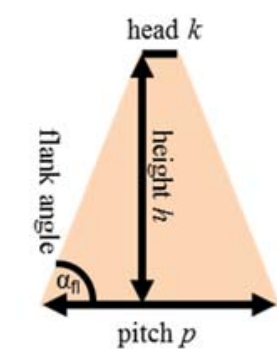

b) flank angle $0^{\circ}$

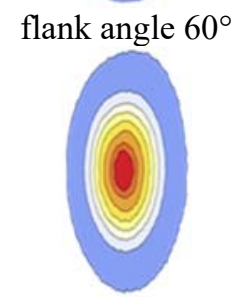

Fig. 1 Projection of the spot area in focal position on a tilted surface, a) 2D-sketch, b) deformation of the laser intensity for different angles of incidence; different projected fluences due to the flank angles

The projected laser fluence $\Phi_{\text {pro }}$ and the flank angle of a tilted surface $\alpha_{\mathrm{fl}}$ is given by

$$
\begin{aligned}
& \Phi_{\text {pro }}=\frac{4 \cdot E_{p}}{\pi \cdot d_{\text {pro }}{ }^{2}} \text { and } \\
& \tan \alpha_{f l}=\frac{h}{1 / 2 \cdot(p-k)},
\end{aligned}
$$

where $E_{p}$ is the laser pulse energy. The projected spot diameter $d_{\text {pro }}$ is the Euclidean distance from the sides of the tilted spot at $1 / \mathrm{e}^{2}$. The decrease of the projected fluence $\Phi_{\text {pro }}$ as a function of the flank angle is given in Fig. 2.

When the ablation threshold, $\Phi_{\text {thres, }}$ is known the maximum possible flank angle $\alpha_{\mathrm{fl}, \max }$ is calculable using standard geometry; it is illustrated in Fig. 2. The achievable height $h$ is determined using Eq. 2 at a given pitch $p$ and a head size $k$.

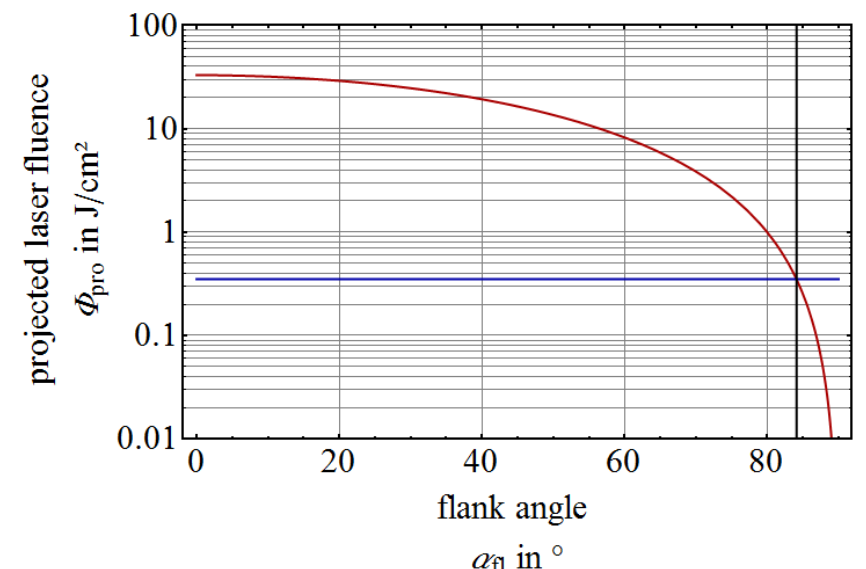

Fig. 2 Decrease of the projected fluence as a function of the flank angle (red). The laser fluence at zero incidence angle is $\Phi=33 \mathrm{~J} / \mathrm{cm}^{2}$ (flank angle $0^{\circ}$ ). The ablation threshold $\Phi_{\text {thres }}$ (blue horizontal) is set to an arbitrary value. Its crossing with local fluence function gives the maximum flank angle (black, vertical).

A simulation of the pyramidal topology with a maximum local fluence of $\Phi_{\text {pro }}=33 \mathrm{~J} / \mathrm{cm}^{2}$ is shown in Fig. 3 . We measured the ablation threshold for sapphire to be $\Phi_{\text {thres }}=680 \pm 60 \mathrm{~mJ} / \mathrm{cm}^{2}$, see Section 3.3. At these conditions a theoretical maximum structure height of $\mathrm{h} \approx 925 \pm 41 \mu \mathrm{m}$ can be achieved. With a pitch of $330 \mu \mathrm{m}$ and a resulting flank of $81.7^{\circ}$ the expected aspect ratio is 3.4. Fabricated sapphire structures are shown in Figure $3 \mathrm{~b}$. The measured flank angle for this sample is $79.1^{\circ}$. We find reasonable agreement between expected and achieved flank angle of the structures.

Due to the chosen structure distance of the pyramids the structure is rounded near the base, such that the top looks like a pyramid while the bottom is more accurately described via a cone topology.
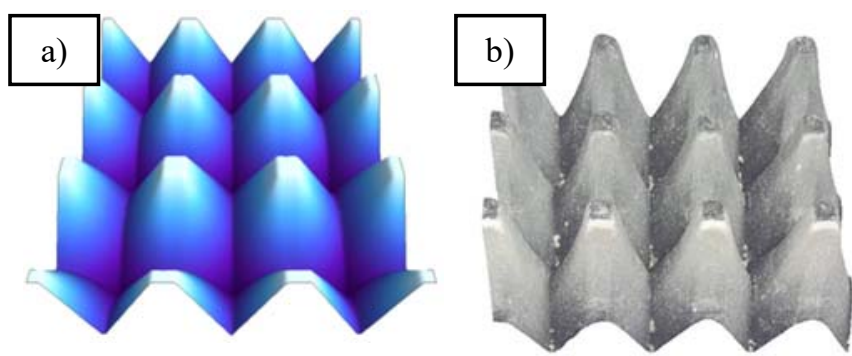

Fig. 3 a) Topology simulation for sapphire; head $k=60 \mu \mathrm{m}$, pitch $p=330 \mu \mathrm{m}$, local fluence $\Phi_{\text {pro }}=33 \mathrm{~J} / \mathrm{cm}^{2}$, ablation threshold $\Phi_{\text {thres }}=0.68 \mathrm{~J} / \mathrm{cm}^{2}$, flank angle $\alpha_{\mathrm{fl}}=81.7^{\circ} ;$ b) optical image stack of a structured sapphire surface, the cones are approx. $700 \mu \mathrm{m}$ high, pitch $p=330 \mu \mathrm{m}$

\subsection{Laser system and beam characteristics}

The laser setup consists of a pico-second laser source with a second harmonic generator $\left(t_{\mathrm{p}} \approx 7 \mathrm{ps}, \lambda=515 \mathrm{~nm}\right.$, $\left.f_{\text {rep }} \leq 400 \mathrm{kHz}, M^{2} \approx 1.04\right)$, and a scanner system. The scanner is equipped with f-theta objective lenses with focal lengths of $255 \mathrm{~mm}$ and $100 \mathrm{~mm}$ to achieve different spot sizes. We measured a laser spot diameter at the sample surface at $1 / \mathrm{e}^{2}$ of $d_{\text {foc }}=30 \mu \mathrm{m}$ and $d_{\text {foc }}=17 \mu \mathrm{m}$ using a Primes MicroSpotMonitor, see Fig. 4. The Rayleigh length $z_{\mathrm{R}}$ was $1.14 \mathrm{~mm}$ and $380 \mu \mathrm{m}$. 


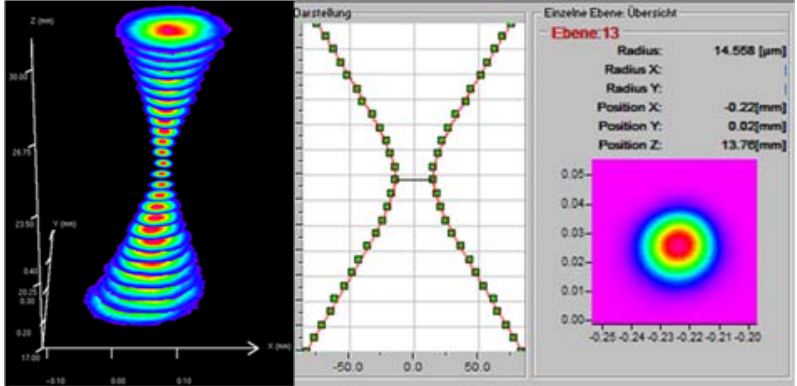

Fig. 4 Beam profile and laser beam caustic

Table 2 Investigated laser parameter range

\begin{tabular}{ccc}
\hline Laser Parameter & Range & Unit \\
\hline Pulse Energy $E_{\mathrm{p}}$ & $5-75$ & $\mu \mathrm{J}$ \\
Repetition rate $f_{\mathrm{rep}}$ & $10-400$ & $\mathrm{kHz}$ \\
Scanning velocity $v$ & $800-6000$ & $\mathrm{~mm} / \mathrm{s}$ \\
z-position multiples of $z_{\mathrm{R}}$ around & $5-7$ & \\
focal position & $1-400$ & \\
Number of passes $N_{\mathrm{p}}$ & \\
\hline
\end{tabular}

\subsection{Laser scanning strategy}

The laser is scanned across the stationary surface $N_{\mathrm{p}}$ times. Each scan pass is a raster pattern giving all ablation lines in the $x$ direction, then all ablation lines in the $y$ direction; see Fig. 5. The depth of the grooves increases with each of the $N_{\mathrm{p}}$ passes.

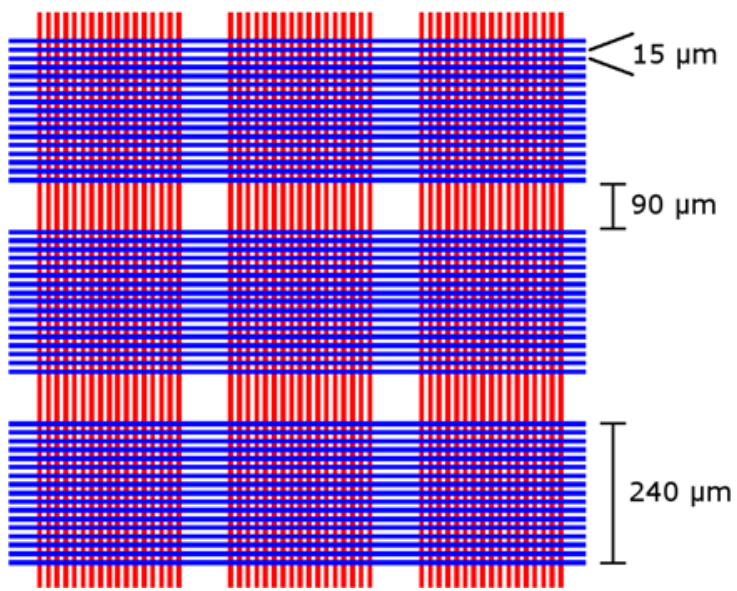

Fig. 5 Scanning strategy with different trajectories (blue and red lines); magnified view

The laser processing time $\tau$ per scan pass for a certain pattern with distinct line length is a function of three factors: the actual laser active time for material processing $\tau_{1}$, the process jump duration for the repositioning of the laser spot for a new scanning line $\tau_{\mathrm{d}}$, and the delay time between the response of the laser system and the scanning device $\tau_{\mathrm{r}}$. This can be written as

$$
\tau=\tau_{l}+\tau_{d}+\tau_{r}
$$

Each of the contributions in Equation 3 is given by

$$
\begin{aligned}
& \tau_{l}=l_{\text {line }} / v_{\text {scan }}, \\
& \tau_{d}=l_{\text {down }} / v_{\text {jump }},
\end{aligned}
$$

where $l_{\text {line }}$ is the length of a single continuous laser ablation process, $l_{\text {down }}$ is the distance between consecutive lines, $v_{\text {scan }}$ is the scan speed, and $v_{\text {jump }}$ is the speed when moving between adjacent lines.

For a total of $N_{\text {lines }}$ per scan path characteristic laserscanner delay time $\tau_{r}$ is given by

$$
\tau_{r}=N_{\text {lines }} \cdot\left(d_{\text {mark }}+d_{\text {lon }}+d_{\text {loff }}\right),
$$

whereas $d$ defines the delay for marking $d_{\text {mark }}$, the laser on delay $d_{\text {lon }}$ and the laser off delay $d_{\text {loff. }}$ We defined an overall area ablation speed $v_{\mathrm{a}}$, which is a useful quantity for scaling processing times from small samples to larger ones

$$
v_{a}=\frac{A}{\tau \cdot N_{p}}
$$

where $A$ is the area of the processed sample.

However, the area speed and the final shape of the SWS depend on the specific ablation parameters including laser power, scan pattern, scan speed, and beam focus position.

\section{Results}

\subsection{Ablation threshold}

We determined the ablation threshold using the procedure described by Liu [13]. We find values of $\Phi_{\text {thres }}=680 \pm 60 \mathrm{~mJ} / \mathrm{cm}^{2}$ and $580 \pm 100 \mathrm{~mJ} / \mathrm{cm}^{2}$ for sapphire and alumina, respectively. At these conditions and with a pitch of $330 \mu \mathrm{m}$ a theoretical pyramid height of $\mathrm{h} \approx 925 \pm 41 \mu \mathrm{m}$ for sapphire and $\mathrm{h} \approx 1013 \pm 90 \mu \mathrm{m}$ for alumina can be achieved. These values are in general agreement with measurements of these samples [14] and in Tab. 3.

The uncertainty in the measured ablation threshold is large compared to other measurements (see for example Gamaly et. al. [15]). We hypothesize that this is due to changes in the opto-thermal properties of the material during the course of ablation. The $17 \%$ uncertainty with alumina could also arise from variation in material uniformity. Further research on this topic is ongoing. We note that away from the focal point the laser beam is slightly deformed, but this effect should not affect the ablation threshold significantly due to slight variation in laser fluence.

\subsection{Pyramid depth vs. repetition rate}

We measured pyramid height as a function of the repetition rate $f_{\text {rep }}$, see Fig. 6, at constant scan passes $N_{\mathrm{p}}=25$. The scanning velocity $v$ has been decreased with increase of the repetition rate to keep the same number of pulses per position $N_{\mathrm{ppp}}$

$$
N_{p p p}=\frac{d_{f o c} \cdot f_{r e p}}{v} .
$$

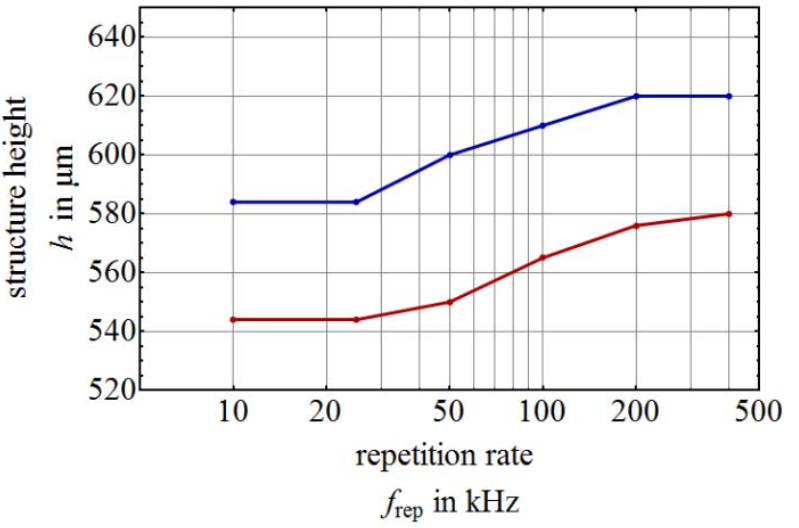

Fig. 6 Structure height as a function of the repetition rate $f_{\text {rep }}$ for alumina (blue) and sapphire (red); $E_{\mathrm{p}}=75 \mu \mathrm{J}, N_{\mathrm{p}}=25, N_{\mathrm{ppp}}=6.8$, $d_{\text {foc }}=17 \mu \mathrm{m}$ processing in focal plane, structure distance $p=330 \mu \mathrm{m}$ 
For all repetition rates the height achieved with alumina is larger by $\sim 40 \mu \mathrm{m}$ compared to sapphire. This result is qualitatively consistent with the difference in ablation thresholds. With both materials, higher repetition rates lead to higher structures. We believe this is due to thermal accumulation between the single laser pulses.

\subsection{Beam focus position}

We assessed the effect of the focus position on the ablation process. The experiments were conducted with a beam size of $17 \mu \mathrm{m}$ and the focus position was varied in increments of Rayleigh length $(380 \mu \mathrm{m})$ from the surface inwards, whereas the surface is set to 0 . We used 4 different pulse energies, and for each pulse energy we used 5 different values of $\tau_{d}$ (see Equation 5). The goal in changing $\tau_{d}$ was to mimic the effect of processing a larger physical area, leading to a larger time delay between two consecutive scans. The results for sapphire are shown in Fig. 7; the results with alumina are similar. The different colored lines represent mimic areas of $1 \mathrm{~mm}^{2}$ (blue line), $13 \mathrm{~mm}^{2}$ (red line), $36 \mathrm{~mm}^{2}$ (green line), $100 \mathrm{~mm}^{2}$ (pink line), and $440 \mathrm{~mm}^{2}$ (gray line) in Fig. 7.

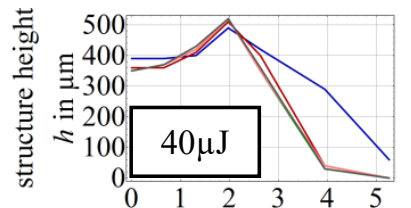

multiples of Rayleigh length

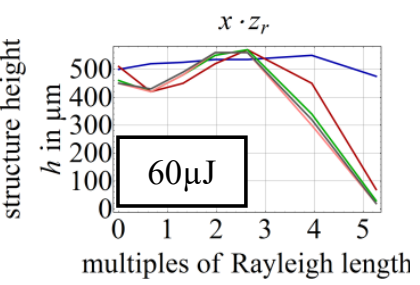

$x \cdot z_{r}$

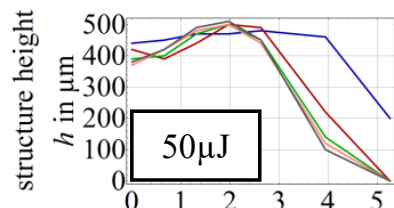

multiples of Rayleigh length

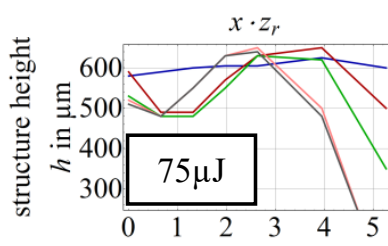

multiples of Rayleigh length $x \cdot z_{r}$
Fig. 7 Sapphire structure height as a function of beam focus position, pulse energy and laser repositioning time $\tau_{d}$, mimic areas with $1 \mathrm{~mm}^{2}$ (blue line), $13 \mathrm{~mm}^{2}$ (red line), $36 \mathrm{~mm}^{2}$ (green line), $100 \mathrm{~mm}^{2}$ (pink line), and $440 \mathrm{~mm}^{2}$ (gray line), Other ablation parameters are $N_{\mathrm{p}}=25, \quad N_{\mathrm{ppp}}=6.8, \quad f_{\text {rep }}=400 \mathrm{kHz}, \quad$ and $d_{\text {foc }}=17 \mu \mathrm{m}$

We observe that for maximum structure height the optimal focus position is generally between 2-4 Rayleigh lengths inside the material and has a weak dependence on pulse energy. It is closer to 4 for the $75 \mu \mathrm{J}$ pulse energy, but closer to 2 for the $40 \mu \mathrm{J}$ at comparative larger structure heights. We ascribe these characteristics to laser processed area dependent heat accumulation, which alters the optical properties of the material. Therefore larger mimic areas have smaller structure heights (gray and pink line in Fig. 7) than smaller mimic areas with more heat accumulation (blue and red line in Fig. 7).

\subsection{Processing and achieved area velocities}

We investigated the dependence of the height of the pyramids on the number of scans $N_{\mathrm{p}}$. Fig. 8 shows the evolution of the ablation up to $N_{\mathrm{p}}=100$, but we used up to $N_{\mathrm{p}}=400$. Fig. 9 gives the resultant geometric measurements.
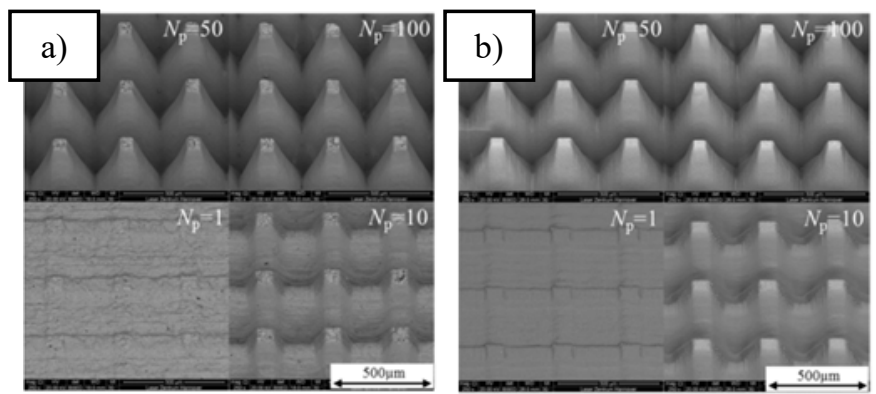

Fig. 8 Laser processed alumina (a) and sapphire (b) as a function of the number of passes $N_{\mathrm{p}}$. For each value of $N_{\mathrm{p}}$ we show an array of $3 \times 3$ pyramids. The other ablation parameters are: $E_{\mathrm{p}}=60 \mu \mathrm{J}, N_{\mathrm{ppp}}=12, f_{\text {rep }}=400 \mathrm{kHz}$, focal position $x \cdot z_{\mathrm{R}}=1$ to the material, and $d_{\text {foc }}=30 \mu \mathrm{m}$ spot size.
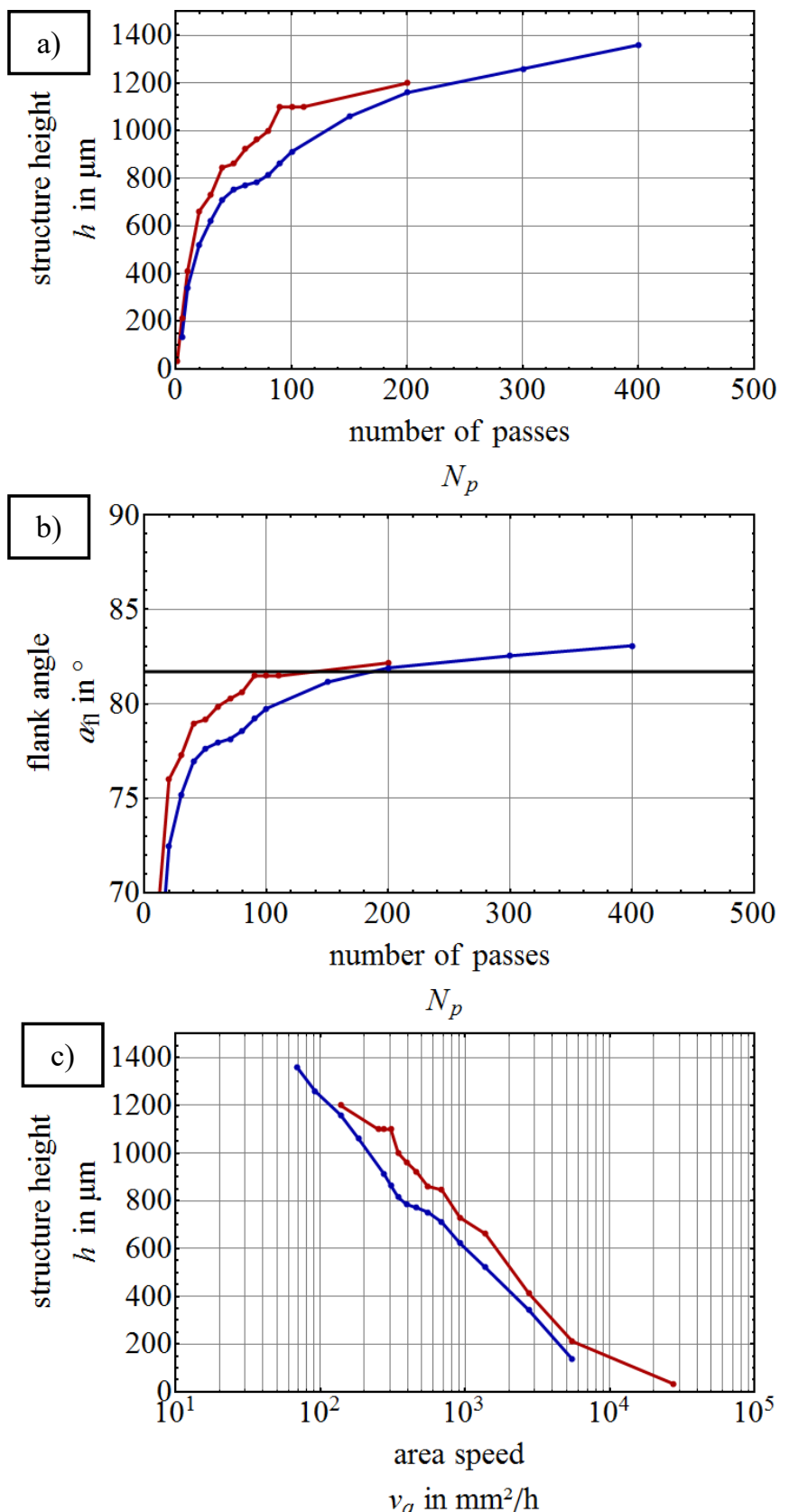

Fig. 9 a) Structure depth as a function of number of passes; b) flank angle as a function of passes; c) Structure depth as a function of area speed; parameters: $E_{\mathrm{p}}=75 \mu \mathrm{J}$, and $f_{\text {rep }}=400 \mathrm{kHz}$ red line $d_{\text {foc }}=30 \mu \mathrm{m}\left(N_{\mathrm{ppp}}=12\right)$, blue line $d_{\mathrm{foc}}=17 \mu \mathrm{m}\left(N_{\mathrm{ppp}}=6.8\right)$; black line in b) theoretical maximum flank angle; focus position was set to 4 times $\mathrm{ZR}$ into the material; used material sapphire 
In Fig. 9 we quantify the evolution of the height with $N_{\mathrm{p}}$. The majority of the ablation is achieved within the first 100 passes. We find that the final flank angle closely matches the predicted value as calculated in Section 2.1 and shown in Fig. 9 b. The structures have rounded edges toward their bases resembling cones. Also, as expected, groove intersections have the deepest depths. The measured structure height is in a good agreement with predictions. We measure a maximum area speed of $v_{\mathrm{a}}=425 \mathrm{~mm}^{2} / \mathrm{h}$ for sapphire and $v_{\mathrm{a}}=405 \mathrm{~mm}^{2} / \mathrm{h}$ for alumina for $1 \mathrm{~mm}$ deep structures with the scan pattern shown in Fig. 2; see Fig. $9 \mathrm{c}$.

Such a high area speed enables the processing of large, $400 \mathrm{~mm}$ diameter, wafers at a stable laser power output in the relatively short time, for this application, of less than two weeks for a sapphire optical element.

\subsection{Transmission spectra of processed samples}

We measured transmission spectra for an alumina and a sapphire sample; see Fig. 10. The transmission measurements and their comparison to predictions are described by Matsumura et al [14]. There is broad agreement between measurements and predictions. The geometrical parameters of the designed and the manufactured samples are given in Tab. 3. The height difference between alumina and sapphire of approximately $15 \%$ is in a good agreement to the difference of the ablation thresholds, whereas the standard deviations are below the estimated value. The standard deviations of the peak width and the pitch are associated on the one hand to the scanning accuracy of the galvo-scanner (2$3 \mu \mathrm{m}$ ), and on the other hand to the opto-thermal change of the material properties during laser processing. Due to boundary considerations, the samples have been full area structured with different strategies. In the case of sapphire the area has been filled with stitched laser processed squares of an edge length of $3.3 \mathrm{~mm}$ and for alumina a continuous laser pattering has been applied with an edge length of $27 \mathrm{~mm}$. Matsumura et al. discuss sources of measurement discrepancies in Fig. 10 in more detail.

Table 3 Design and achieved geometric parameters of the structured samples

\begin{tabular}{cccc}
\hline Sample & $\begin{array}{c}\text { Height } \\
\text { in } \mu \mathrm{m}\end{array}$ & $\begin{array}{c}\text { Pitch } \\
\text { in } \mu \mathrm{m}\end{array}$ & $\begin{array}{c}\text { Peak width } \\
\text { in } \mu \mathrm{m}\end{array}$ \\
\hline \multicolumn{4}{c}{ Designed } \\
All & 810 & 330 & 60 \\
\hline \multicolumn{4}{c}{ Measured } \\
Alumina & $790 \pm 60$ & $313 \pm 4$ & $66 \pm 8$ \\
Sapphire & $715 \pm 24$ & $325 \pm 4$ & $57 \pm 6$ \\
\hline
\end{tabular}

Further, the simulation has been extended up to $350 \mathrm{GHz}$ to assess the broadband performance of the laser machined surface topology. In result, the reflectivity is less than $3 \%$ from $170-300 \mathrm{GHz}$ for both polarizations and both materials. A total bandwidth of $130 \mathrm{GHz}$ is achieved with band averaged reflectivity of $1.5 \%$ [14].
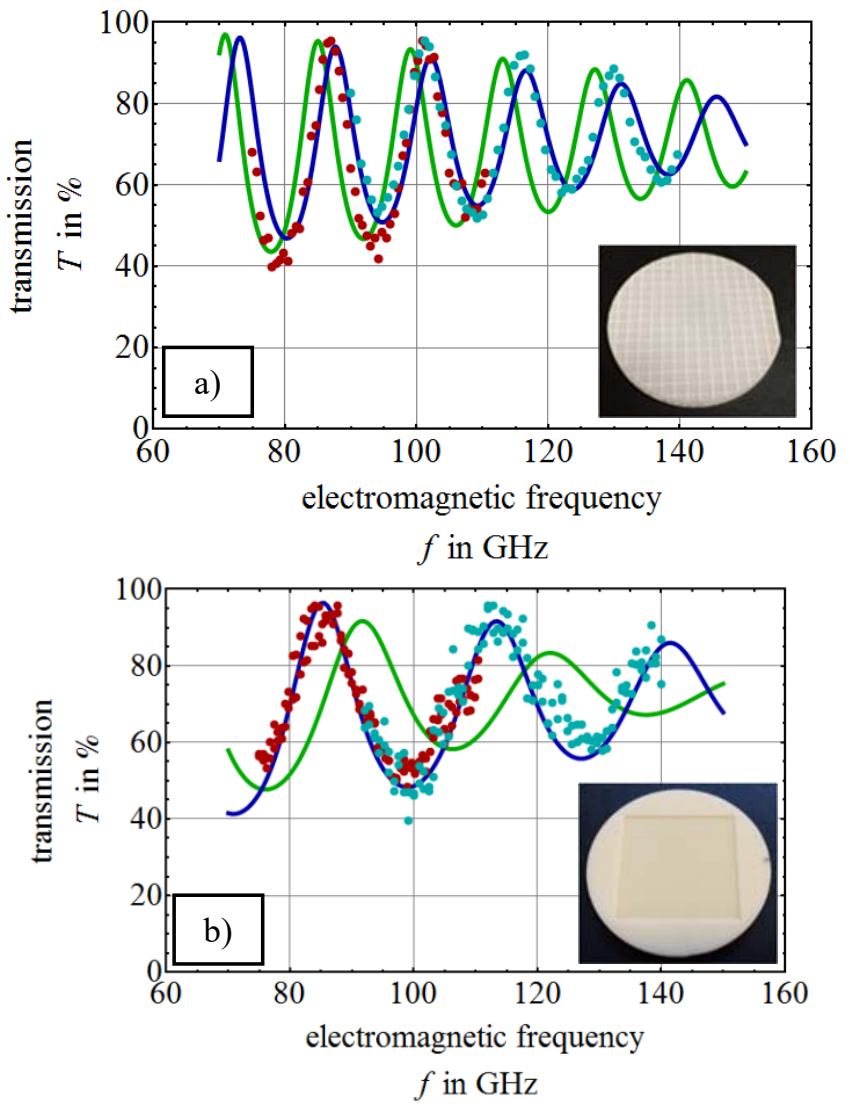

Fig. 10 Transmittance as a function of frequency; blue line prediction for the smallest structures, green prediction for the tallest structures; red and cyan dots measured transmittance spectra; a) Sapphire with photo inlet of the sample and b) Alumina with photo inlet of the sample; [data taken from 14]

\section{Summary and outlook}

We demonstrated that laser ablation is a suitable tool to machine mm-size SWS on alumina and sapphire, which are otherwise difficult to process. Such structures can serve as effective broad band anti-reflection coatings in the $\mathrm{mm}$ wave band of frequencies. The presented model is able to predict the height of the structures, and we find agreement with the fabricated structures. The dependence of the ablation on pulse energy, repetition rate, and beam focus position has been demonstrated. We find that beam focus position is particularly important, because the structure dimensions depend on the surface irradiating beam size and energy as far as 4 times the Rayleigh lengths away from the focal position. This is associated with the time dependent changes of the opto-thermal properties of the material during laser processing.

The area speeds achieved are sufficiently fast that substrates of several hundred $\mathrm{mm}$ diameter can be fabricated within a reasonable amount of time. Such optical elements are now being used by experiments designed to measure the cosmic microwave background radiation.

Finally, we find that transmission spectra of samples ablated with SWS structures agree with predictions, demonstrating that SWS structures are an appealing approach for making anti-reflection coatings in the mm-wave range. 


\section{Acknowledgements}

The authors acknowledge use of resources provided by the Minnesota Nanofabrication Center (http://nfc.umn.edu), the University of Minnesota Imaging Center (http://uic.umn.edu), and the Minnesota Supercomputing Institute (http://www.msi.umn.edu).

The authors would also like to thank to Prof. M. Hasegawa for us to use the millimeter wave source.

This work was partially supported by JSPS KAKENHI Grant Number 70625003, the Mitsubishi foundation (grant number 24 in JFY2015 in science and technology) and the ISAS strategic development fund from the steering committee for space science.

\section{References}

[1] M. Niemack and ACTPol Collaboration: American Astronomical Society, AAS Meeting, (2013) 221-105.04.

[2] K. MacDermid, A.M. Aboobaker, P. Ade, F. Aubin, C. Baccigalupi, K. Bandura, C. Bao, J. Borrill, D. Chapman, J. Didier, M. Dobbs, J. Grain, W. Grainger, S. Hanany, K. Helson, S. Hillbrand, G. Hilton, H. Hubmayr, K. Irwin, B. Johnson, A. Jaffe, T. Jones, T. Kisner, J. Klein, A. Korotkov, A. Lee, L. Levinson, M. Limon, A. Miller, M. Milligan, E. Pascale, K. Raach, B. Reichborn-Kjennerud, C. Reintsema, I. Sagiv, G. Smecher, R. Stompor, M. Tristram, G. Tucker, B. Westbrook, and K. Zilic: Proc. SPIE, 9153, (2014) 915311.

[3] B.A. Benson, P. Ade, Z. Ahmed, S. Allen, K. Arnold, J. Austermann, A. Bender, L. Bleem, J. Carlstrom, C. Chang, H. Cho, J. Cliche, T. Crawford, A. Cukierman, T. de Haan, M. Dobbs, D. Dutcher, W. Everett, A. Gilbert, N. Halverson, D. Hanson, N. Harrington, K. Hattori, J. Henning, G. Hilton, G. Holder, W. Holzapfel, K. Irwin, R. Keisler, L. Knox, D. Kubik, C. Kuo, A. Lee, E. Leitch, D. Li, M. MacDonald, S. Meyer, J. Montgomery, M. Myers, T. Natoli, H. Nguyen, V. Novosad, S. Padin, Z. Pan, J. Pearson, C. Reichardt, J. Ruhl, B. Saliwanchik, G. Simard, G. Smecher, J. Sayre, E. Shirokoff, A. Stark, K. Story, A. Suzuki, K. Thomson, C. Tucker, K. Vanderlinde, J. Vieira, A. Vikhlinin, G. Wang, V. Yefremenko, and K. Yoon: Proc. SPIE, 9153, (2014) 91531P.

[4] A. Suzuki, P. Ade, Y. Akiba, C. Aleman, K. Arnold, M. Atlas, D. Barron, J. Borrill, S. Chapman, Y. Chinone, A. Cukierman, M. Dobbs, T. Elleflot, J. Errard, G. Fabbian, G. Feng, A. Gilbert, W. Grainger, N. Halverson, M. Hasegawa, K. Hattori, M. Hazumi, W. Holzapfel, Y. Hori, Y. Inoue, G. Jaehnig, N. Katayama, B. Keating, Z. Kermish, R. Keskitalo, T. Kisner, A. Lee, F. Matsuda, T. Matsumura, H. Morii, S. Moyerman, M. Myers, M. Navaroli, H. Nishino, T. Okamura, C. Reichart, P. Richards, C. Ross, K. Rotermund, M. Sholl, P. Siritanasak, G. Smecher, N. Stebor, R. Stompor, J. Suzuki, S. Takada, S. Takakura, T. Tomaru, B. Wilson, H. Yamaguchi, and O. Zahn: J. Low Temperature Physics, 176, (2014) 719.

[5] W. H. Southwell: J. Opt.Soc. America A: Optics, Image Science, and Vision, 8, (1991) 549.

[6] R. Datta, C. D. Munson, M. D. Niemack, J. J. McMahon, J. Britton, E. J. Wollack, J. Beall, M. J. Devlin, J. Fowler, P. Gallardo, J. Hubmayr, K. Irwin, L. Newburgh, J. P. Nibarger, L. Page, M. A. Quijada, B. L.
Schmitt, S. T. Staggs, R. Thornton, and L. Zhang: Appl. Opt., 52, (2013) 8747.

[7] H. Tran and L. Page: J. Physics: Conference Series, 155, (2009) 012007.

[8] W. J. Tropf and M. E. Thomas: "Handbook of Optical Constants of Solids" ed. by E. D. Palik, (Publisher Academic Press, 1998) p.676.

[9] B. Chichkov, C. Momma, S. Nolte, F. von Alvensleben, and A. Tünnermann: J. Appl. Phys. A, 63, (1996) 109.

[10]F. Dausinger, H. Hügel, and V. Konov: Proc. ALT-02, Advanced Laser Technologies, Adelboden, (2002)

[11]S. Nolte, C. Momma, H. Jacobs, A. Tünnermann, B. Chichkov, B. Wellegehausen, and H. Welling: J. Opt. Soc. Am. B., 14, (1997) 2716.

[12] V. Schütz, U. Stute, and A. Horn: Physics Procedia, 41, (2013) 633.

[13]J.M. Liu: Opt. Lett., 7, (1982) 196.

[14]T. Matsumura, K. Young, Q. Wen, S. Hanany,

H. Ishino, Y. Inoue, M. Hazumi, J. Koch, O. Suttmann, and V. Schütz: Applied Optics, 55, (2016) in press.

[15]E. G. Gamaly, N. R. Madsen, M. Duering, A. V. Rode, V. Z. Kolev, and B. Luther-Davies: Phys. Rev. 71, (2005) 174405 .

(Received: January 29, 2016, Accepted: April 25, 2016) 